\title{
Cyber Physical Autonomous Mobile Robot (CPAMR) Framework in the Context of Industry 4.0
}

\author{
Yoon Ket Lee ${ }^{1, a}$, Yeh Huann Goh ${ }^{1}$ and Yiqi Tew ${ }^{2}$ \\ ${ }^{1}$ Faculty of Engineering, Tunku Abdul Rahman University College, Kuala Lumpur, Malaysia \\ ${ }^{2}$ Faculty of Computing and Information Technology, Tunku Abdul Rahman University College, Kuala Lumpur, \\ Malaysia
}

\begin{abstract}
Industry 4.0 or Smart Manufacturing creates intelligent object networking and independent process management through Internet of thing and data services. Cyber-Physical System (CPS) communicates among humans, machines and products through Internet of Things (IoT). In this paper a cyber physical autonomous mobile robot (CPAMR) IoT infrastructure system has been proposed. It is capable of performing human-machine interact by allowing users to place and manage orders using cloud platform. The Enterprise Resource Planning (ERP) system processes the data and send the product's data to Radio-frequency identification (RFID) tag system for storage and printout. Through Remote Telemetry Unit (RTU), the status of the product, CPAMR system and workstations or machineries are linked to the cloud platform. Initially, system identify the locations of CPAMR, product and the desired workstation. After gathering all the required information, Artificial Intelligence Algorithms (AIA) performs real time route map planning according to the shortest distance between CPAMR and the destination. This route map planning will then be sent to the CPAMR's microcontroller for operation. While the CPAMR is moving, it moves according to the planned route map with the assistance of the Obstacle Avoidance System until it reaches the destination and notifies cloud platform. Three individual projects representing three main functions of the proposed CPAMR have been carried out. Results show that the framework of the project is viable.
\end{abstract}

\section{Introduction}

Industry 4.0 or Smart Manufacturing is one of the hottest topics in the industry and become the world trend in the industry evolution [1-2]. Industry 4.0 concept is driven by the German whereas smart manufacturing leads by America. Both industry concepts are relatively similar. Industry 4.0 represents the coming fourth industrial revolution on the way to an Internet of thing, data services. Decentralized intelligence helps create intelligent object networking and independent process management, with the interaction of the real and virtual worlds representing a crucial new aspect of the manufacturing and production process [3]. One of the key factors to the success of implementing Industry 4.0 is through the Cyber-Physical System (CPS). CPS communicates among humans, machines and products with the help of Internet of Things (IoT). CPS system is able to acquire and process data, this

\footnotetext{
${ }^{a}$ Corresponding author: leeyk@tarc.edu.my
} 
feature allows the CPS system to perform self-control in completing certain task and interact with humans via interfaces [4].

In an ideal production world, mass production with single product range and long product cycle life will definitely achieve the lowest product cost per unit. However in reality this is not feasible due to mass production, if the company continuously produce products with regardless of the sale demand from the market then it will end up keeping the stock in the warehouse even though the cost per production is low but the holding cost and deprecation cost of the products will end up a disaster to the company. Furthermore it almost impossible that has a good demand from market with only single produce range and long product cycle life. So the optimum solution to this is to increase the flexibility in production line but yet in the mass production environment. Flexibility in an automatic manufacturing system is always a challenge to the users or researchers. In order to achieve the desire flexibility, one of the solutions is through the mobile material handling system. It has to move independently to transport goods or process workpieces. It also need to movable lightweight robots to fully autonomously operating systems. In production shops, mobile robots have to be using modern navigation technique to move entirely without the need for floor markings, induction loops or magnets. It has to be real time application to deal with the any changes in production line. If production line has the mix product schedule, this Artificial Intelligence Algorithms (AIA) for the CPAMR can arrange the route map of the product according to the virtual world requirement, current product, machinery and also environment status.

In this research proposal framework, we investigate the usage of a novel CPS system in a smart material handling system. The proposed CPS system is equipped with the features of IoT, intelligent vision system and Autonomous Mobile Robot (AMR). The proposed Cyber-Physical AMR (CPAMR) system is embedded with AIA which allows the CPARM system to do decision making on production schedule planning, machinery, transportation requirement from production environment, work order for a part from virtual and part status based on the virtual and physical world interaction.

\section{Related Work}

An extensive literature reviews on several papers have been studied as categorizes below:

\subsection{IoT and CPS Architecture}

Lee et al. proposed 5-level CPS structure 5C, which consist of Connection, Conversion, Cyber, Cognition and Configure [4]. This 5C provides a viable and practical guideline for manufacturing industry to implement CPS for better product quality and system reliability with more intelligent and resilient manufacturing equipment. Gigli et al. make a comment on the IoT architecture that there still do not have the standard to put together different types of technologies such as sensors, actuators, network communications and computing technologies [5]. There are different IoT architecture models that have been proposed by the researchers. The architecture recommended by the International Telecommunication Union (ITU) has 5 layers which are Sensing Layer, Access Layer, Network Layer, Middle-ware Layer and Application Layers. This proposed IoT layer is similar to the Open Systems Interconnection (OSI) model for network and data communication. Qian et al. stated that traditional IoT structure is formed by three layers Perception Layer (responsible for recognizing and collecting information), Transportation Layer (consists of data communication networks) and Application Layer (applications which rely on the data from perception layer) [6]. Xu et al. suggested a collective summary of recommended architectures by different researchers which is similar to the structure proposed by the ITU above [7]. This proposed solution takes a service-oriented architecture (SOA) approach which is normally deployed in business software systems. The four-layer architecture is Sensing Layer, Networking Layer, Service Layer and Interface Layer. Trappey et al. proposed Perception layer, Transmission layer, Computation layer, Application layer in the IoT architecture [8].

The authors found that the above proposed IoT architecture even though there same to be different and every researcher try to have their own standard but the basic functionality and concept does not differ very much. 


\subsection{Autonomous Mobile Robot Device}

A Propose AMR is a material handling device that consists of two major components. The moving component is an AGV and the material handling components is a robotic arm. The robotic arm is mounted onto the AGV. The type of robotic arm is flexible, it depends on the production line requirements however the mobility is fixed where it require the AMR to transport the robotic arm from one destination to another destination. Therefore the authors decide to review AGV research only. There are extensive researches have been conducted by the researchers on the topic of AGV. These papers can be further classified into the short summary below:

\subsubsection{Localization Techniques}

Jones suggested ideas on line following AGV, where the robot are able to localize itself once the AGV reaches a node or intersection of lines [9]. Tsumura and Markosov et al. found that Unable to localize AGVs along the lines, only at nodes [10-11] AGVs unable to travel without line, and unable to travel back to the line once its off-course. Require smooth surface for implementations. Lawrence proposed ideas of localization through odometry and Inertial Navigation System. However this method suffers from: Accumulative errors due to wheel slip. Unable to use the system for long period of time [12]. Highly dependent on modelling of system where many parameters needed to be considered such as slip, coefficient of friction, power draw from drivetrain, etc. Bosien et al. proposes the usage of RFID tags that are planted around the environment in the form of an array [13]. Thus allowing robots to localize depending on which RFID it is able to scan. The paper did not suggest a method to improve its accuracy, because by detecting RFID, there is a range that will produce inconsistency of results that lead to the pose of the robots cannot be accurately determined. Farkas et al. used triangulation method in their paper and propose the usage of Wi-Fi access points and the signal strength of the access points to determine the position of the AGV [14].

\subsubsection{Docking Techniques}

Lee et al. AGV maneuver and docking system based on bird's eye view image extraction [15]. The system used Hough line transform the estimate the distance between markers and the AGV itself. The testing data shows high efficiency and accuracy while traveling. This does not depend of fix line to travel to destinations. However does not include object avoidance system. Andersson et al. presents use of dead reckoning along with computer vision [16]. The AGV maneuver's along a preprogrammed path by using dead reckoning and odometry localization techniques. Computer vision is responsible for picking up images of markers located on the floor. However AGV suffers from accumulative errors due to the usage of odometry localization. Kim et al. published a paper regarding the implementation of object tracking with visual docking for performing tasks with multiple AGVs joining together [17]. The AGV's docking was done with 2 steps. Approaching and docking, where the first phase uses computer vision to track another AGV and determine the trajectory towards the $\mathrm{AGV}$. The second phase starts when the AGVs are required to dock on another AGV using vision as well as with the help of compass sensors for heading and range finders to determine trajectory path. The testing of AGV docking system was done on surface of water and require high power processing unit due to requirement of high level of control of AGV. Vaz et al. presented the usage of infra-red sensing with known reflective panels strategically placed to allow localization of AGV [18]. This paper demonstrates high accuracy for the AGV to know where the docking area is but AGV may only be able to localize with the presence of the infra-red lasers only.

From the literature review we found that the paper presented by [15] research is very close to one of the research objectives or the function that we looking for [15] approach is a real time and flexible route map planning to the need of the requirement. However there is a major drawback of the system that the system does not include object avoidance systems. The feature is extremely important, during the traveling of the AGV, obstacle may appear randomly this will disturb the AGV performance or even end the process therefore in this proposal we should include the obstacle avoidance system. 
Apart from this, the proposed system also need to include the AIA for object recognition to identify the destiny and to perform CPAMR route map planning.

\subsection{Obstacle Avoidance System for (AGV)}

Though out the literature review, we find that there are three types of obstacle avoidance system for an AGV, they are non-vision based, vision-based and sensor fusion based obstacle avoidance system.

\subsubsection{Non-vision based}

Arboleda et al. use simple obstacle avoidance system which only using ultrasonic or sonar sensor to detect obstacle is insufficient because sonar sensor has limitation in detecting obstacle surface which is not smooth or rigid [19]. Not effective when there are slopes, slants, and rough surfaces in front of AGV where the Sonar sensors might result in false readings Requires. Freitas et al. proposed use laser scanner for obstacle avoidance system [20]. Effective for rough surfaces, slants and slopes but only limited to certain range of coverage.

\subsubsection{Vision based}

Polanczyk et al proposed using stereo camera with the algorithm consists of three stages which are the camera motion estimation, building a map of obstacles and path planning [21]. Preliminary obstacle detection is carried based on the stereo-vision depth image. The ambient obstacles are searched in parallel with camera motion estimation. Points that are located at distances larger than 10 meters from the camera are discarded as disparity for a closer point can be estimated more accurately. On the other hand, the points within the range of 0.8 meters from the camera are considered to be an obstacle and taken into consideration for further construction of the map. This method is effective for any kind of surface but limited Region of Interest (ROI) depending of the Field of View (FOV) of the camera.

\subsubsection{Sensor Fusion based}

Monteiro et al. proposed system applied both Laser Range Finder and the camera are mounted in a common base where both sensors axes are arranged in parallel and aligned ideally [22]. The calibration is necessary to obtain a mapping expression to transform the points in the laser reference system to the camera reference system and then to image plane. Heavy algorithms yet integration of sensors system is required after sensors calibration.

A convincing conclusion can be drawn from literature review that this proposed CPAMR is feasible. There are no existing work have been conducted by researchers in this area. But yet there are feasible research has been proposed by the existing researcher individually. The authors integrating the existing research work by proposing adopting [7]. IoT Architecture in the project since this model is collective summary of different researchers and normally deployed in business system. Beside that, model is adopted for Real time and Flexible route map planning system and for the vision-based model will be selected for the obstacle avoidance system.

\section{Proposed CPAMR System}

The authors propose this CPAMR framework has 3 Basic core functions as shown in the Figure 1, 2 and 3. Whereby the communication between the real and virtual by applying IoT framework through the RFID sensing, RTU, Cloud, MQTT protocol and ERP platform. Besides, through the RFID sensing, the autonomous system or AMR can identify the process required by the cloud platform then the CPAMR is capable to perform the real time route map planning and obstacle avoidance system for the independent process management. 


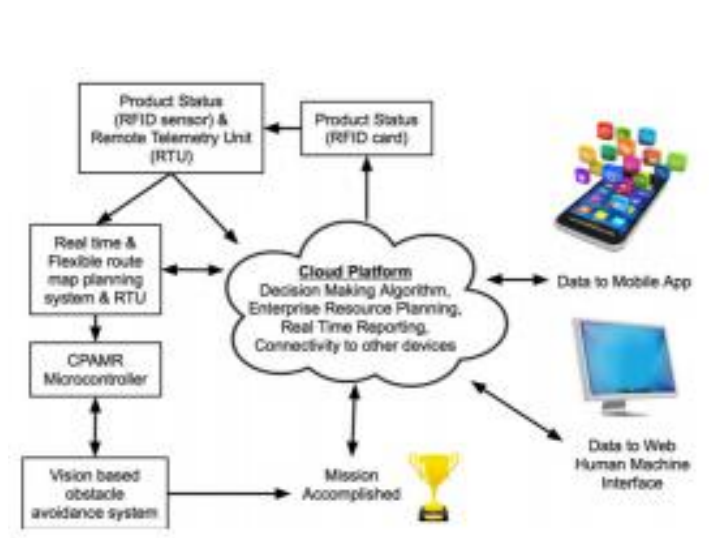

Fig. 1. Proposed CPAMR's IoT infrastructure framework

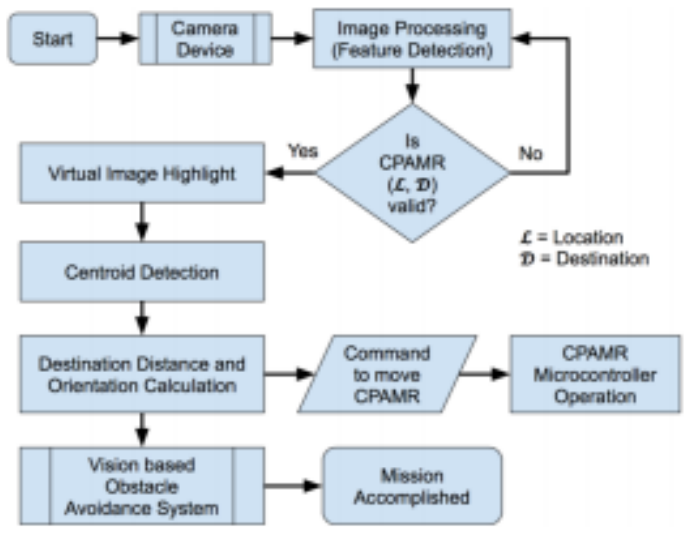

Fig. 2. Route Map Planning Flow Chart

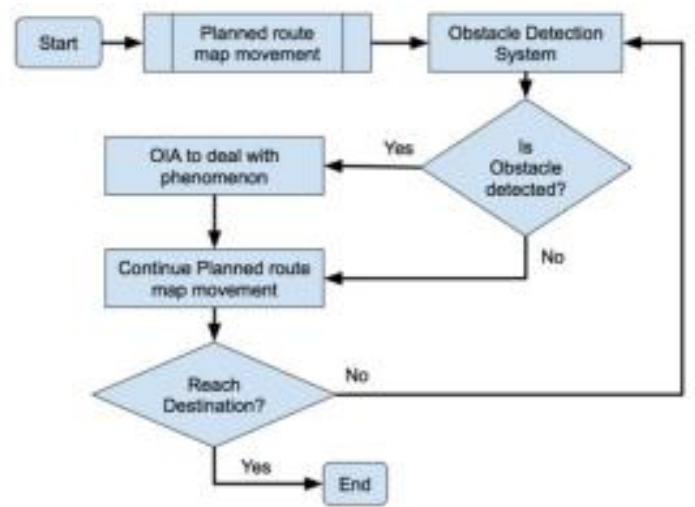

Fig. 3. Vision based Obstacle Avoidance System Flow Chart

\subsection{System Platform}

A CPAMR's IoT infrastructure system is developed in this study. Fig. 1 shows the proposed CPAMR's IoT infrastructure framework. Once the clients post the order and the requirements from the mobile or WEB based HMI to the cloud, the ERP system will process the data and send the product's data to RFID tag system for storage and printout. The RFID tag adhere to the product and send to the production line. Through Remote Telemetry Unit (RTU), the status by the product, CPAMR system and workstations or machineries are linked to the cloud platform. When the product is released to the production line, the RFID sensor will detect the product through the RFID tag. It will request the CPAMR to pick the product and transport to the corresponding workstation that required by the product. Before the CPAMR move, the cloud will notify the real time and Flexible route map planning system to start the route map planning as show in the Fig. 2. When the system starts, it will identify the CPAMR location, product location and the desired workstation. Once the location image is identified, the virtual image will be highlighted to extract the figure for centroid detection. AIA will calculate the shortest distance and orientation route map planning for the CPAMR. This route map planning will then sent to the CPAMR's micro-controller for operation. CPAMR mobile moves toward the destination according to the received road map. While the CPAMR is moving, the obstacle avoidance system will turn on as shown in Fig. 3. When it detects the obstacle, the AIA will trigger and deal with the phenomenon. CPAMR has to continue the planed route map until it reaches the destination and notify cloud platform. 


\subsection{System Testbed}

This proposed CPAMR is a massive research work which requires different area of expertise for instances IoT, real time planned AMR and image processing for obstacle avoidance system. The authors divided the whole project into 3 project groups which each of the FYP students will conduct the initial study in the similar area of this research work. For the IOT project, Amazon Web Service platform has been employed as a base for center hub, the module like Amazon S3, Amazon DynamoDB and Amazon SNS is being employed for the IOT processing part. In term of connectivity and data control, protocol Message Queue Telemetry Transport (MQTT), RFID sensor and tags, Arduino, Esspressolite V 2.0 (RTU) have been used to build prototype. Real Time Route Map Planned AMR, a web camera, mechanical structure, motor, Raspberry Pi, Arduino, motor driver, Esspressolite V 2.0 and MQTT protocol have been engaged to construct the AMR. For the obstacle avoidance system, a series of imaging processing technique has been applied to develop AIA to avoid the obstacle during the moving. Imaging processing technique like Raw RGB Image captured, Converted Gray-scale and Blurred Image, Canny Edge Detection, Binary Image with Segmented Regions with Noise, Filtered and Eroded Binary Image with Segmented Region, Binary Image with Center of Obstacle Detected have been applied to develop the AIA [23].

\section{Results and Discussion}

This primary research project has been conducted under the Faculty of Engineering, Tunku Abdul Rahman University College. The 3 primary functions of CPAMR feasibility study has been investigated by 3 different undergraduate FYP students. The primary results of the investigations are positive. A student has achieved the fundamental function of IOT framework by using Amazon Web Service package and MQTT protocol to communicate between the modules to acquire data from the field and send the signal to the field, a student demonstrated using the web camera to identify the location of AGV and destination, by applying image processing algorithms the student is able to plan the route map for the AGV and pass over the command to micro controller (Arduino) through MQTT protocol to control the AMR to move to the destination on real time random route map planning basis. Meanwhile another student also successfully demonstrated the control of the AGV to avoid the obstacles during the moving. These primary results are very encouraging and also proven the CPAMR framework concept is viable. Further research and development challenge is on how to integrate these 3 different projects into a master project. Parameters and performance measures of the CPAMR need to be defined to suit the Industry 4.0 research.

\section{Conclusion}

On the whole, the initial study of the CPAMR demonstrate the framework of the project is viable, the three main functionality of the proposed CPAMR are being achieved at the basic level from different project group. Although it is beginning stage of the research project, it shows a good start to a final massive project which requires expertise from various area such as IoT, Big data analytic, autonomous system, manufacturing planning and control, data security and etc.

\section{References}

1. McKinsey Digital, "Industry 4.0 after initial hype", https://www.mckinsey. de/ files/mckinsey industry 40 2016.pdf, 2016, [Accessed on 31 July 2017].

2. R. Geissbauer, S. Schrauf, V. Koch, S. Kuge, "Industry 4.0 - Opportunities and Challenges of the Industrial Internet," PricewaterhouseCoopers Aktiengesellschaft Wirtschaftsprufungsgesellschaft (PwC) ", pp. 1-52, December 2014.

3. W. MacDougall, "Industrie 4.0 Smart Manufacturing for the future," Germany Trade and Invest, pp. 1-40, July 2014. 
4. J. Lee, B. Bagheri, H-A. Kao, "A Cyber-Physical Systems architecture for Industry 4.0-based manufacturing systems," Manufacturing letter, vol.3, pp.18-23, January 2015.

5. M. Gigli, S. Koo, "Internet of Things: Services and Application Categorization," Advances in Internet of Things, vol.1, pp.27-31, July 2011.

6. X. Qian, J. Zhang, "Study on the structure of Internet of Things (IOT) business operation support platform," IEEE International Conference on Communication Technology (ICCT), pp.1068-1071, January 2011.

7. L. D. Xu, W. He, S. Li, "Internet of Things in Industries: A Survey," IEEE Transactions on Industrial Informatics, vol.4, pp.2233-2243, January 2014.

8. A. J. C. Trappey, C. V. Trappey, U. H. Govindarajan, A. C. Chuang, J. J. Sun, "A review of essential standards and patent landscapes for the Internet of Things: A key enabler for Industry 4.0," Advanced Engineering Informatics, IN PRESS, December 2016.

9. J. L. Jones, A. M. Flynn, B. A. Seiger, "Mobile Robots: Inspiration to Implementation, Second Edition" CRC Press, April 1993.

10. T. Tsumura, "Survey of autonomous guided vehicles in japanese factory", IEEE International Conference on Robotics and Automation, pp.1329-1334, April 1986.

11. D. Markosov, H. Durrant-Whyte, "Mobile Vehicle Navigation in Unknown Environments: Multiple Hypothesis Approach", IEE Proceedings of Control Theory nad Applications, vol.142(4). pp.385-400, Jul 1995.

12. A. Lawrence, "Modern Inertial Technology: Navigation, Guidance, and Control," Springer Science \& Business Media, September 2001.

13. A. Bosien, M. Venzke, V. Turau, "A rewritable RFID environment for AGV navigation", International Workshop on Intelligent Transportation, March 2008.

14. K. Farkas, A. Huszak, G. G ' odor, "Optimization of Wi-Fi Access Point ' Placement for Indoor Localization”. Journal Informatics \& Information Technology Today, vol.1(1), pp.28-33, July 2013.

15. J. Lee, C-H. Hyun, M. Park, "A Vision-Based Automated Guided Vehicle System With Marker Recognition for Indoor Use,” Sensors, vol.13(8), pp.10052-10073, August 2013.

16. D. Andersson, E. Mansson, "Positioning and Docking of an AGV in a Clinical Environment" Master's thesis in Biomedical Engineering, Chalmers University of Technology, 2012.

17. Y-H. Kim, S-W. Lee, H. S. Yang and D. A. Shell, "Toward Autonomous Robotic Containment Booms: Visual Servoing for Robust inter-Vehicle Docking for Surface Vehicles," Intelligent Service Robotics, vol.5(1). pp.1-18, January 2012.

18. P. M. Vaz, R. Ferreira, V. Grossman, M. I. Ribeiro, "Docking of Mobile Platform based on Infrared Sensors," IEEE International Symposium on Industrial Electronics, pp.735-740, July 1997.

19. E. R. Arboleda, M. C. T. Alegre, K. F. Idica, "Development of a Low Cost Electronic Wheelchair with obstacle Avoidance Feature," Journal of Mechatronics, Electrical Power and Vehicular Technology, vol.06, pp.89- 96, December 2015.

20. G. Freitas, B. Hamner, M. Bergerman, S. Singh, “A Practical Obstacle Detection System for Autonomous Orchard Vehicles," International Conference on Intelligent Robots and Systems, pp.3391-3398, October 2012.

21. M. Polanczyk, M. Strzelecki, K. ' Slot, "Obstacle Avoidance Procedure ' and Lee Algorithm Based Path Replanner for Autonomous Mobile Platform," International Journal of Electronics and Telecommunications, vol.59(1), pp.85-91, March 2013.

22. G. Monteiro, C. Premebida, P. Peixoto, U. Nunes, "Tracking and Classification of Dynamic Obstacles Using Laser Range Finder and Vision," International Conference on Intelligent Robots and Systems, pp.1-7, 2006.

23. YK Lee, JM Lim, KS Eu, YH Goh, Y Tew," Real Time Image Processing Based Obstacle Avoidance and Navigation System for Autonomous Wheelchair Application," 2017 Asia-Pacific Signal and Information Processing Association Annual Summit and Conference (APSIPA ASC),accepted, December 2017 